\title{
The Graceful Coalescence of Alpha Cycles
}

\author{
Christian Barrientos $^{1 *}$, Sarah Minion²
}

\begin{abstract} for this type of subdivided graphs.

Keywords: Coalescence, $\alpha$-labeling, Graceful labeling, Ladder.

2010 AMS: Primary 05C78, Secondary 05C76

${ }^{1}$ Department of Mathematics, Valencia College, Orlando, Florida, USA, ORCID: 0000-0003-2838-8687

${ }^{2}$ Department of Mathematics, Valencia College, Orlando, Florida, USA, ORCID: 0000-0002-8523-3369

*Corresponding author: chr_barrientos@yahoo.com

Received: 30 December 2018, Accepted: 21 February 2019, Available online: 27 June 2019
\end{abstract}

The standard coalescence of two graphs is extended, allowing to identify two isomorphic subgraphs instead of a single vertex. It is proven here that any succesive coalescence of cycles of size $n$, where $n$ is divisible by four, results in an $\alpha$-graph, that is, the most restrictive kind of graceful graph, when the subgraphs identified are paths of sizes not exceeding $\frac{n}{2}$. Using the coalescence and another similar technique, it is proven that some subdivisions of the ladder $L_{n}=P_{2} \times P_{n}$ also admit an $\alpha$-labeling, extending and generalizing the existing results

\section{Introduction}

A difference vertex labeling of a graph $G$ of size $n$ is an injective mapping $f$ from $V(G)$ into a set $M$ of nonnegative integers, such that every edge $u v$ of $G$ has assigned a weight defined by $|f(u)-f(v)|$. All labelings considered in this work are difference vertex labelings. A labeling is called graceful when $M=\{0,1, \ldots, n\}$ and the induced weights are $1,2, \ldots, n$. If $G$ admits such a labeling, it is called a graceful graph. Let $G$ be a bipartite graph where $\{A, B\}$ is the natural bipartition of $V(G)$, we refer to $A$ and $B$ as stable sets of $V(G)$. A bipartite labeling of $G$ is an injection $f: V(G) \rightarrow\{0,1, \ldots, t\}$ for which there exists an integer $\lambda$, named the boundary value of $f$, such that $f(u) \leq \lambda<f(v)$ for every $(u, v) \in A \times B$, that induces $n$ different weights. This is an extension of the definition given by Rosa and Šráň [1]. From the definition we conclude that the labels assigned by $f$ on the vertices of $A$ and $B$ are in the interval $[0, \lambda]$ and $[\lambda+1, t]$, respectively. When $t=n$, the function $f$ is an $\alpha$-labeling and $G$ is an $\alpha$-graph.

Let $f: V(G) \rightarrow\{0,1, \ldots, t\}$ be a labeling of a graph $G$ of size $n \leq t$. The labeling $g: V(G) \rightarrow\{c, c+1, \ldots, c+t\}$, defined for every $v \in V(G)$ and $c \in \mathbb{Z}$ as $g(v)=c+f(v)$, is the shifting of $f$ in $c$ units. Note that this labeling preserves the weights induced by $f$.

If $f$ is bipartite with boundary value $\lambda$, the labeling $h: V(G) \rightarrow\{0,1, \ldots, t+d-1\}$, defined for every $v \in V(G)$ and $d \in \mathbb{Z}^{+}$ as $h(v)=f(v)$ when $f(v) \leq \lambda$ and $h(v)=f(v)+d-1$ when $f(v)>\lambda$, is the bipartite d-labeling of $G$ obtained from $f$. This labeling uses labels from $[1, \lambda] \cup[\lambda+d, t+d-1]$. In other terms, this labeling shifts the weights induced by $f$ in $d-1$ units. Thus, if $f$ is an $\alpha$-labeling of $G$ and $d$ is a positive constant, then $h$ is a $d$-graceful labeling. This concept was introduced, independently by Maheo and Thuillier [2] and Slater [3] in 1982.

In the following sections we study $\alpha$-labelings of the coalescence of $\alpha$-cycles. Suppose that $G_{1}$ and $G_{2}$ are two graphs such that $H$ is an induced subgraph of both of them. The $H$-coalescence, or simply coalescence, of $G_{1}$ and $G_{2}$, denoted by $G_{1} \cdot G_{2}$, is the graph obtained by identifying the copy of $H$ in $G_{1}$ with the copy of $H$ in $G_{2}$. Assuming that for $i=1,2$, the graph $G_{i}$ has 
order $n_{i}$ and size $m_{i}$, and $H$ has order $p$ and size $q$, then $G_{1} \cdot G_{2}$ has order $n_{1}+n_{2}-p$ and size $m_{1}+m_{2}-q$. In Figure 1.1 we show an example of this operation where $G_{1}$ and $G_{2}$ are isomorphic and $H \cong C_{4}$, which is represented in the picture with green edges.

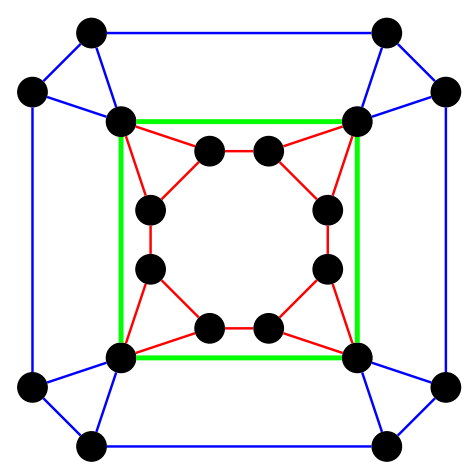

Figure 1.1. The $C_{4}$-coalescence of two isomorphic graphs of order 12 and size 20

In [4], Barrientos proved that if $H \cong P_{1}$ and $G_{1}$ and $G_{2}$ are $\alpha$-graphs, there is a coalescence (also named one-point union or vertex amalgamation) of them is an $\alpha$-graph. In [5], Barrientos and Minion showed that if $H \cong P_{2}$ and $G_{1}$ and $G_{2}$ are $\alpha$-graphs, the coalescence ( or edge amalgamation) of them is an $\alpha$-graph if the edge of minimum weight in $G_{1}$ is identified with the edge of maximum weight of $G_{2}$. In this article, we extend the idea of the edge amalgamation, presented in [5], considering $H$ to be a path. All graphs considered here are finite with no loops or multiple edges. We use the notation and terminology used in [6] and [7].

\section{Preliminary results}

In his seminal paper, Rosa [8] showed that when $n \equiv 0(\bmod 4)$, there exists an $\alpha$-labeling of the cycle $C_{n}$. We present here two labelings of $C_{n}$ that are going to be used in the proof of the main result of the next section.

Suppose that $V\left(C_{n}\right)=\left\{v_{1}, v_{2}, \ldots, v_{n}\right\}$ is the vertex set of $C_{n}$ and its edge set is $\left\{v_{i} v_{i+1}: 1 \leq i \leq n\right\}$ where the addition is taken modulo $n$. The labelings $f$ and $g$ given below are two well-known $\alpha$-labelings of $C_{n}$. The interested reader can easily verify this statement.

$$
\begin{aligned}
& f\left(v_{i}\right)= \begin{cases}\frac{i-1}{2} & \text { if } i \text { is odd and } 1 \leq i \leq \frac{n}{2}-1, \\
\frac{i+1}{2} & \text { if } i \text { is odd and } \frac{n}{2}+1 \leq i \leq n-1, \\
n+1-\frac{i}{2} & \text { if } i \text { is even. }\end{cases} \\
& g\left(v_{i}\right)= \begin{cases}\frac{i-1}{2} & \text { if } i \text { is even and } 2 \leq i \leq \frac{n}{2}-1, \\
\frac{i}{2} & \text { if } i \text { is even and } \frac{n}{2}+2 \leq i \leq n, \\
n-\frac{i-1}{2} & \text { if } i \text { is odd. }\end{cases}
\end{aligned}
$$

In Figure 2.1 we show two examples for each of these labelings. The graphs on the first row are labeled using the function $f$, while $g$ is used to label the graphs on the second row. The arrow inside the cycle shows the orientation of the vertices within each graph.

\section{Graceful coalescence}

Let $C_{n_{1}}, C_{n_{2}}, \ldots, C_{n_{k}}$ be a collection of cycles, where the vertex set is $V\left(C_{n_{j}}\right)=\left\{v_{1, j}, v_{2, j}, \ldots, v_{n_{j}, j}\right\}$ and the edge set is $E\left(C_{n_{j}}\right)=\left\{v_{1, j} v_{2, j}, v_{2, j} v_{3, j}, \ldots, v_{n_{k}-1, j} v_{n_{k}, j}, v_{n_{k}, j} v_{1, j}\right\}$ for each $j \in\{1,2, \ldots, k\}$. For every $j \in\{1,2, \ldots, k-1\}$, select a positive integer $t_{j}$ such that $2 t_{j} \leq \min \left\{n_{j}, n_{j+1}\right\}$. A graph $G$ is a coalescence of the cycles $C_{n_{1}}, C_{n_{2}}, \ldots, C_{n_{k}}$ when the vertices $v_{1, j+1}, v_{2, j+1}, \ldots, v_{t_{j}, j+1}$ of $C_{n_{j+1}}$ (together with the induced edges) are identified with the vertices $v_{n_{j}-t_{j}+1, j}, v_{n_{j}-t_{j}+2, j}, \ldots v_{n_{j}, j}$ of $C_{n_{j}}$, respectively. Note that $G$ is a graph of order

$$
\sum_{j=1}^{k} n_{j}-\sum_{j=1}^{k-1} t_{j}=n_{k}+\sum_{j=1}^{k-1}\left(n_{j}-t_{j}\right)
$$



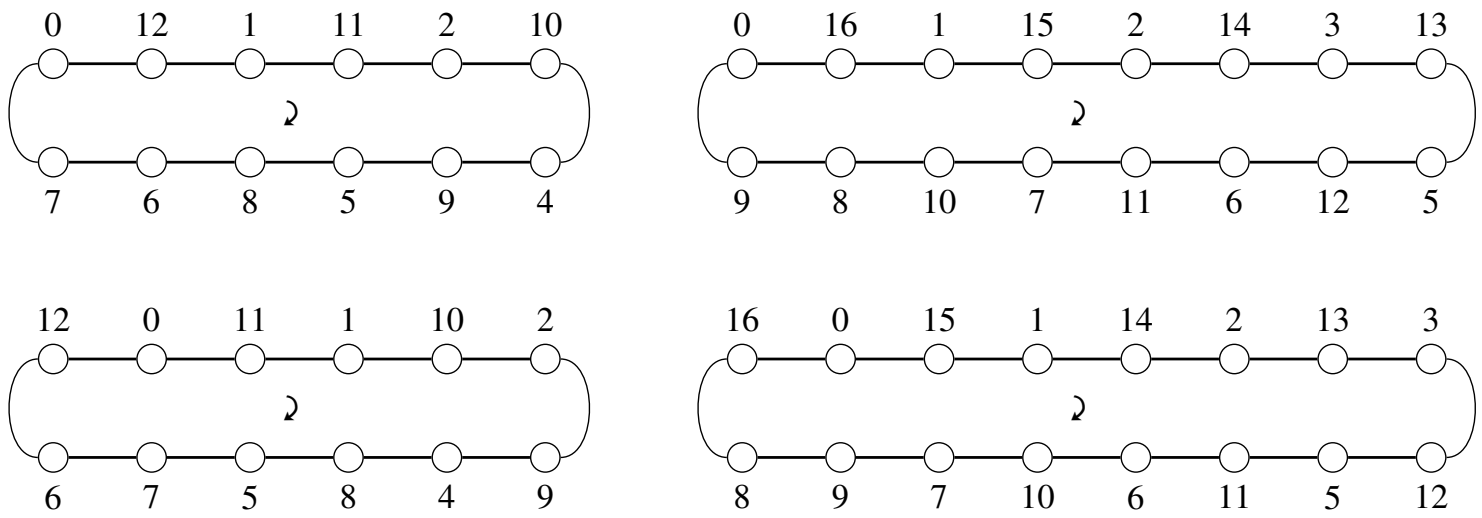

Figure 2.1. Two $\alpha$-labelings of $C_{12}$, and $C_{16}$

and size

$$
\sum_{j=1}^{k} n_{j}-\sum_{j=1}^{k-1}\left(t_{j}-1\right)=n_{k}+\sum_{j=1}^{k-1}\left(n_{j}-t_{j}+1\right)
$$

In Figure 3.1 we show an example of this construction where $n_{1}=8, n_{2}=12, n_{3}=12, n_{4}=8$, and $n_{5}=4$, and $t_{1}=3$, $t_{2}=4, t_{3}=4$, and $t_{4}=2$. The numbers inside each cycle correspond to their respective vertices within that cycle.

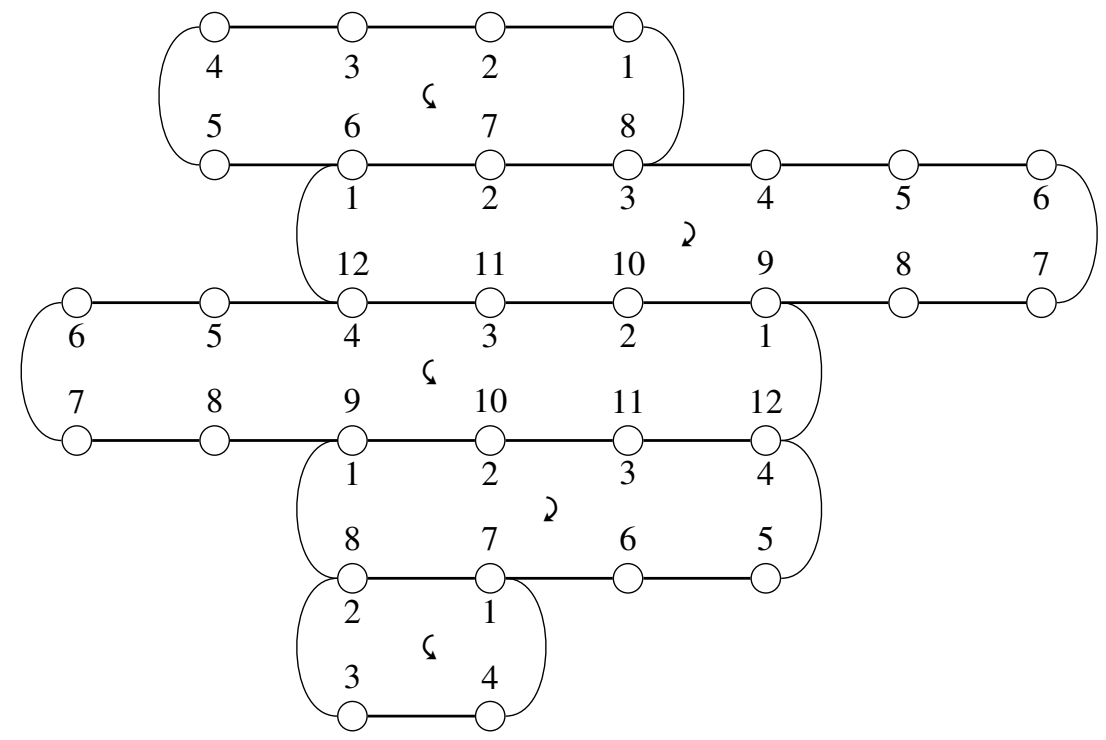

Figure 3.1. A coalescence of $C_{8}, C_{12}, C_{12}, C_{8}$ and $C_{4}$

We claim that when each $n_{j} \equiv 0(\bmod 4)$, the coalescence $G$ of the cycles $C_{n_{1}}, C_{n_{2}}, \ldots, C_{n_{k}}$, determined by $t_{1}, t_{2}, \ldots, t_{k-1}$, is an $\alpha$-graph. Within the proof of this theorem we use the labelings $f$ and $g$ of $C_{n}$ given in Section 2.

Theorem 3.1. Let $G$ be the coalescence of the cycles $C_{n_{1}}, C_{n_{2}}, \ldots, C_{n_{k}}$ determined by the integers $t_{1}, t_{2}, \ldots, t_{k-1}$, where $2 t_{j} \leq \min \left\{n_{j}, n_{j+1}\right\}$. If for every $\left.j \in\{1,2, \ldots, k\}, n_{j} \equiv 0(\bmod 4)\right\}$, then $G$ is an $\alpha$-graph.

Proof. Let $G$ be the coalescence of the cycles $C_{n_{1}}, C_{n_{2}}, \ldots, C_{n_{k}}$ determined by the integers $t_{1}, t_{2}, \ldots, t_{k-1}$, where, for every $j \in\{1,2, \ldots, k\}, 2 t_{j} \leq \min \left\{n_{j}, n_{j+1}\right\}$ and $\left.n_{j} \equiv 0(\bmod 4)\right\}$. Thus, every $C_{n_{j}}$ admits an $\alpha$-labeling.

To start, we label the vertices of $C_{n_{1}}$ using the labeling $f$. For each $j \in\{2,3, \ldots, k\}$, the selection of the initial labeling used on the vertices of $C_{n_{j}}$ depends on the labeling used on $C_{n_{j-1}}$, according to the following criteria: 
- If $t_{j-1}$ is even, both $C_{n_{j-1}}$ and $C_{n_{j}}$ have the same type of labeling.

- If $t_{j-1}$ is odd, $C_{n_{j-1}}$ and $C_{n_{j}}$ have different types of labelings.

Now that every cycle $C_{n_{j}}$ has been $\alpha$-labeled, we proceed to modify these initial labelings to obtain the desired $\alpha$-labeling of $G$.

Recall that for every $j \in\{1,2, \ldots, k\}$, the size of the coalescence of the cycles $C_{n_{1}}, C_{n_{2}}, \ldots, C_{n_{k}}$ determined by the integers $t_{1}, t_{2}, \ldots, t_{k-1}$, is

$$
\sum_{i=j}^{k} n_{i}-\sum_{i=j}^{k-1}\left(t_{i}-1\right)=n_{k}+\sum_{i=j}^{k-1}\left(n_{i}-t_{i}+1\right)
$$

where the term $\sum_{i=j}^{k-1}\left(t_{i}-1\right)$ is the number of edges shared by $C_{n_{j}}$ and $C_{n_{j+1}}$.

The $\alpha$-labeling of $C_{n_{j}}$ is transformed into a $d_{j}$-graceful labeling (the intermediate labeling), where

$$
d_{j}=\left(1+n_{k}+\sum_{i=j}^{k-1}\left(n_{i}-t_{i}+1\right)\right)-n_{j}
$$

In this way, the weights on the edges of $C_{n_{j}}$ form the interval

$$
I_{j}=\left[n_{k}+n_{k}+\sum_{i=j}^{k-1}\left(n_{i}-t_{i}+1\right)-\left(n_{j}-1\right), n_{k}+\sum_{i=j}^{k-1}\left(n_{i}-t_{i}+1\right)\right] .
$$

Since $\min \left\{I_{j}: 1 \leq j \leq k\right\}=1$ and $\max \left\{I_{j}: 1 \leq j \leq k\right\}=n_{k}+\sum_{i=1}^{k-1}\left(n_{i}-t_{i}+1\right)$, that is, the size of $G$, we get

$$
\bigcup_{j=1}^{k} I_{j}=\left[1, n_{k}+\sum_{i=1}^{k-1}\left(n_{i}-t_{i}+1\right)\right]
$$

Now, we need to shift these labelings to perform the coalescence of the labeled cycles. The labels assigned to the vertices of $C_{n_{1}}$ constitute the final labeling of this cycle. For every $j \in\{2,3, \ldots, k\}$, the final labeling of $C_{n_{j}}$ is obtained recursively in the following manner:

Assume that the labeling of $C_{n_{j-1}}$ is its final labeling. Let $L_{j-1}$ be the set of the labels assigned to the vertices shared by $C_{n_{j-1}}$ and $C_{n_{j}}$. The final labeling of $C_{n_{j}}$ is obtained by adding the constant $\min L_{j-1}$ to every label of $C_{n_{j}}$. Thus, the only overlapping of vertex labels between $C_{n_{j-1}}$ and $C_{n_{j}}$ occurs on the vertices used to produce the coalescence.

Once this process has been completed, we have a bipartite labeling of $G$ where the induced weights are $1,2, \ldots, n_{k}+$ $\sum_{j=1}^{k-1}\left(n_{j}-t_{j}+1\right)$, with no label repeated.

Therefore, $G$ is an $\alpha$-graph.

In Figure 3.2 we show the final $\alpha$-labeling of the coalescence of the cycles $C_{16}, C_{12}, C_{8}, C_{12}, C_{8}$, determined by the integers $t_{1}=5, t_{2}=3, t_{3}=4, t_{4}=3$. The starting $\alpha$-labelings of the cycles are: $(0,16,1,15,2,14,3,13,5,12,6,11,7,10,8,9),(12$, $0,11,1,10,2,9,4,8,5,7,6),(0,8,1,7,3,6,4,5),(0,12,1,11,2,10,4,9,5,8,6,7),(8,0,7,1,6,3,5,4)$. The intermediate $d$-graceful labelings are: $(0,45,1,44,2,43,3,42,5,41,6, \mathbf{4 0}, \mathbf{7}, \mathbf{3 9}, \mathbf{8}, \mathbf{3 8}),(\mathbf{3 3}, \mathbf{0}, \mathbf{3 2}, \mathbf{1}, \mathbf{3 1}, 2,30,4,29, \mathbf{5}, \mathbf{2 8}, \mathbf{6}),(\mathbf{0}, \mathbf{2 3}, \mathbf{1}$, $22,3,21,4,20),(0,18,1,17,2,16,4,15,5,14,6,13),(8,0,7,1,6,3,5,4)$. The shifting constants are $7,12,15$, and 21, respectively. The highlighted numbers correspond to the vertices that are going to be identified to produce the graph $G$.

Suppose that we want to form all nonisomorphic coalescence graphs constructed with $k$ copies of $C_{n}$, where $n \equiv 0(\bmod 4)$. How many of these graphs exist? Since two consecutive copies of $C_{n}$ shared at most $\frac{n-2}{2}$ edges, any graph obtained by the coalescence of these cycles can be described by a sequence (or string) of numbers from $\left\{1,2, \ldots, \frac{n-2}{2}\right\}$. Thus, counting nonismorphic coalescence graphs is equivalent to count nonoriented strings with $k-1$ beads of $\frac{n-2}{2}$ or fewer colors. This number is known and can be found in OEIS A277504 [9]. In the following table we show the first values for $n \in\{4,8,12,16,20\}$ and $k \in\{1,2, \ldots, 11\}$.

In Figure 3.3 we show the $\alpha$-labelings of the six graphs obtained using three copies of $C_{8}$. 


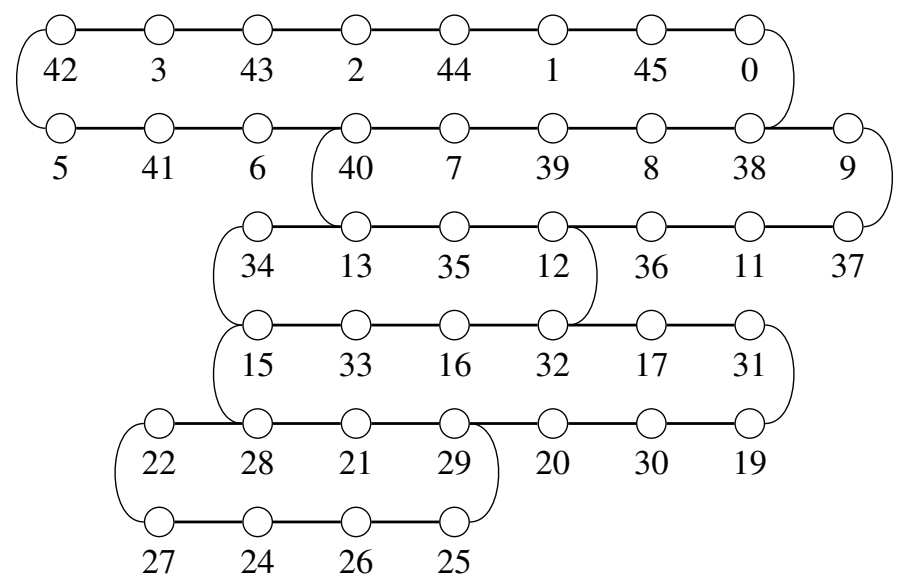

Figure 3.2. $\alpha$-labeling of a coalescence of cycles

\begin{tabular}{|r|r|r|r|r|r|}
\hline$k \backslash n$ & 4 & 8 & 12 & 16 & 20 \\
\hline 1 & 1 & 1 & 1 & 1 & 1 \\
\hline 2 & 1 & 3 & 5 & 7 & 9 \\
\hline 3 & 1 & 6 & 15 & 28 & 45 \\
\hline 4 & 1 & 18 & 75 & 196 & 405 \\
\hline 5 & 1 & 45 & 325 & 1225 & 3321 \\
\hline 6 & 1 & 135 & 1625 & 8575 & 29889 \\
\hline 7 & 1 & 378 & 7875 & 58996 & 266085 \\
\hline 8 & 1 & 1134 & 39375 & 412972 & 2394765 \\
\hline 9 & 1 & 3321 & 195625 & 2883601 & 21526641 \\
\hline 10 & 1 & 9963 & 978125 & 20185207 & 193739769 \\
\hline 11 & 1 & 29643 & 4884375 & 141246028 & 1743421725 \\
\hline
\end{tabular}

Table 1. Number of nonisomorphic coalescence graphs formed with $k$ copies of $C_{8}$

\section{Graceful subdivision of ladders}

In this section we present two graceful labelings of subdivisions of ladders; the first result is a corollary of Theorem 3.1, the second one is a new construction. The ladder $L_{n}$ is the result of the Cartesian product of the paths $P_{2}$ and $P_{n}$. The edges of $P_{2}$ within $L_{n}=P_{2} \times P_{n}$ are called the steps of $L_{n}$. This type of graph can be seen as the coalescence of $n-1$ copies of $C_{4}$, therefore, $L_{n}$ is an $\alpha$-graph. The fact that $L_{n}$ is graceful was proven first by Acharya and Gill [10].

In a graph $G$, a subdivision of an edge $u v$ is the operation of replacing $u v$ with a path $u, w, v$ through a new vertex $w$. If the edhe $u v$ is replaced with the path $u, w_{1}, w_{2}, \ldots, w_{t}, v$, we say that $u v$ has been subdivided an even (resp. odd) number of times when $t$ is even (resp. odd). Kathiresan [11] has shown that graphs obtained from ladders by subdividing each step exactly once are graceful.

If every step of $L_{n}$ is subdivided an even number of times, then two consecutive subdivided steps, together with the two edges connecting them, form a cycle of size divisible by 4. Using Theorem 3.1, we can prove that this type of subdivided ladder is a graceful graph; in fact, it is an $\alpha$-graph.

Corollary 4.1. If every step of the ladder $L_{n}=P_{2} \times P_{n}$ is subdivided an even number of times, the resulting graph is an $\alpha$-graph.

In Figure 4.1 we show, together with the original labeling of $L_{5}$, two examples of this subdivided ladder.

Unfortunately, the argument used in this corollary does not work when the edges are subdivided an odd number of times. So it is an open problem to find an $\alpha$-labeling or a graceful labeling of these subdivided ladders. 

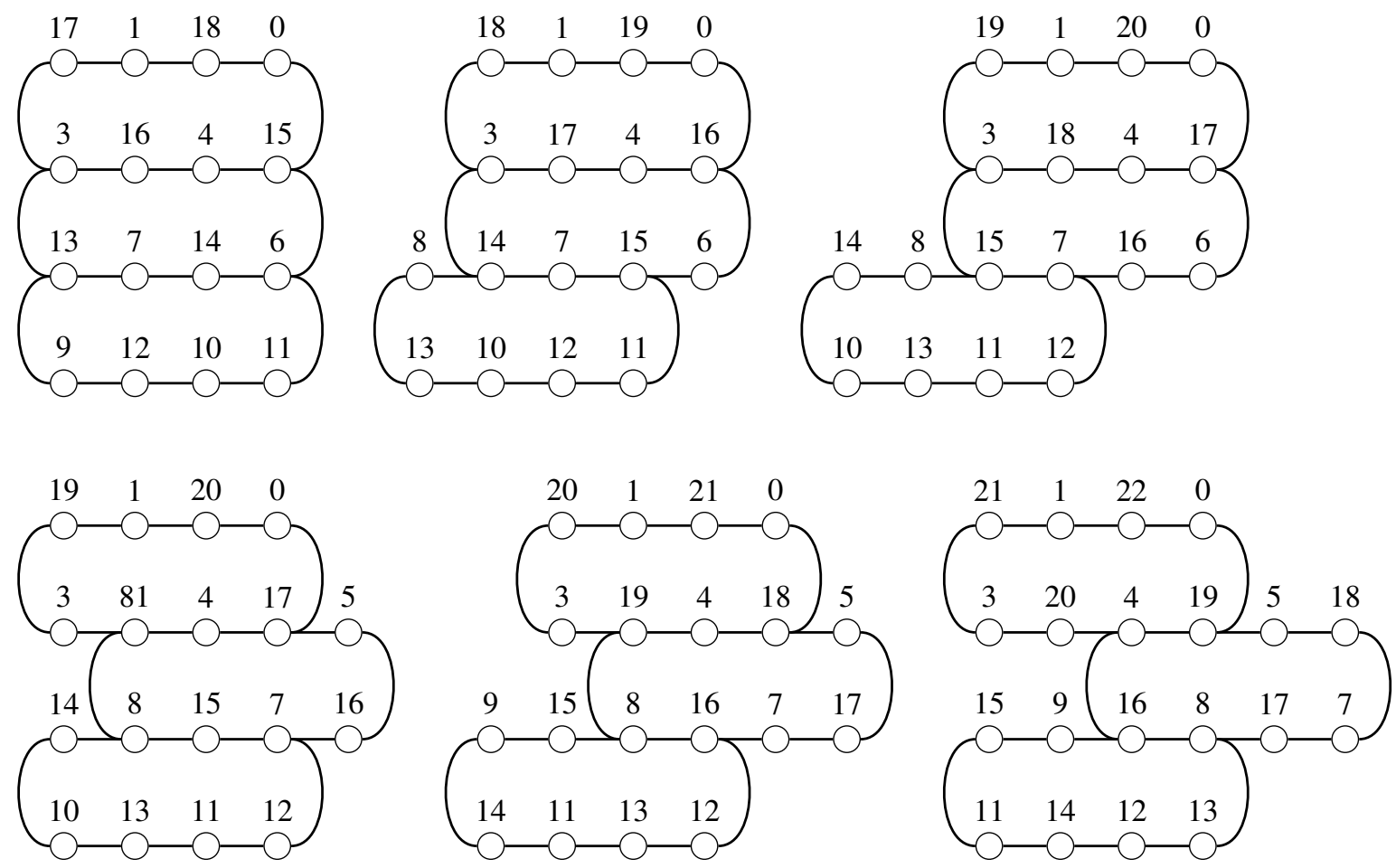

Figure 3.3. $\alpha$-labelings of all the coalescences of three copies of $C_{8}$
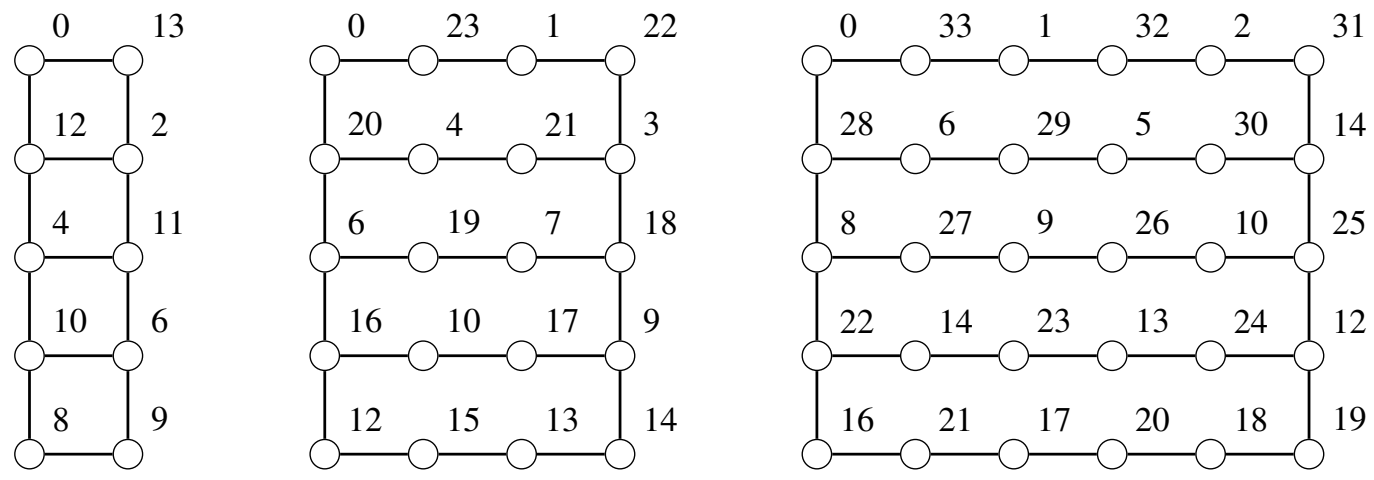

Figure 4.1. $\alpha$-labeling of the ladder $L_{5}$ and some of its even subdivisions

Now we turn our attention to the graph obtained by subdividing every edge of $L_{n}$ exactly once. We claim that this graph admits an $\alpha$-labeling. Even when the resulting graph can be seen as the coalescence of $n-1$ copies of $C_{8}$, we use here a different construction based on the facts that $C_{8}$ and $P_{5}$ are $\alpha$-graphs. The basic labelings of these graphs are given below:

- For $C_{8}$, the consecutive labels are: 5, 0, 8, 1, 7, 3, 6, 4 .

- For $P_{5}$, the consecutive labels are: 2, 1, 3, 0, 4 .

Suppose that $G_{n}$ denotes the graph obtained by subdividing once all the edges of $L_{n}$. When $n=2, G_{2} \cong C_{8}$; we use on $G_{2}$ the $\alpha$-labeling given above. To obtain an $\alpha$-labeling of $G_{3}$ we transform the $\alpha$-labeling of $G_{2}$ by shifting its weights in such a way that the new largest label is $14=8+6$, that is, the size of $G_{2}$ plus 6 . The labeling of $P_{5}$ is shifted $\lambda_{2}+2$ units, where $\lambda_{2}$ is the boundary value of the $\alpha$-labeling of $G_{2}$. The vertices $\lambda_{2}-1$ and $\lambda_{2}$ in $G_{2}$ are connected to the vertices to the vertices $\lambda_{2}+4$ and $\lambda_{2}+6$ in $P_{5}$; thus, the new edges have weights 5 and 6 , respectively. The resulting graph is $G_{3}$ with an $\alpha$-labeling. We continue this process until $G_{n}$ has been labeled. In this way we have proved the following theorem. 
Theorem 4.2. The graph $G$, obtained by subdividing every edge of the ladder $L_{n}$ exactly once, is an $\alpha$-graph.

In Figure 4.2 we show the first four cases of this construction.
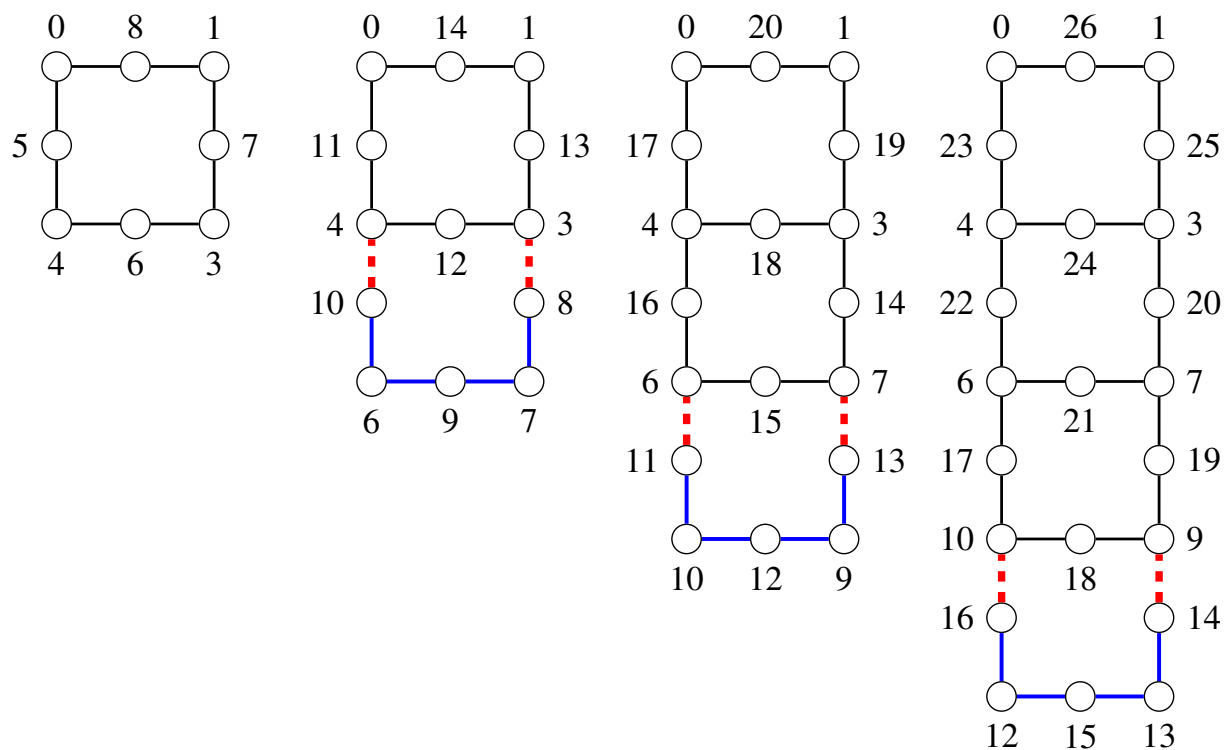

Figure 4.2. $\alpha$-labelings of subdivided ladders

\section{References}

[1] A. Rosa, J. Širáň, Bipartite labelings of trees and the gracesize, J. Graph Theory, 19 (1995), 201-215.

[2] M. Maheo, H. Thuillier, On d-graceful graphs, Ars Combin., 13 (1982), 181-192.

[3] P. J. Slater, On k-graceful graphs, Proceedings of the 13th Southeastern International Conference on Combinatorics, Graph Theory and Computing, (1982), 53-57.

[4] C. Barrientos, Graceful labelings of chain and corona graphs, Bull. Inst. Combin. Appl., 34 (2002), 17-26.

[5] C. Barrientos, S. Minion, Alpha labelings of snake polyominoes and hexagonal chains, Bull. Inst. Combin. Appl., 74 (2015), 73-83.

[6] G. Chartrand, L. Lesniak, Graphs \& Digraphs, 2nd ed. Wadsworth \& Brooks/Cole, 1986.

[7] J. A. Gallian, A dynamic survey of graph labeling, Electronic J. Combin., 21(\#DS6), 2018.

[8] A. Rosa, On certain valuations of the vertices of a graph, Theory of Graphs (International Symposium, Rome, July 1966), Gordon and Breach, N. Y. and Dunod Paris (1967), 349-355.

[9] J.-F. Sloane, https://oeis.org/A277504, 2016.

[10] B. D. Acharya, M. K. Gill, On the index of gracefulness of a graph and the gracefulness of two-dimensional square lattice graphs, Indian J. Math., 23 (1981), 81-94.

[11] K. Kathiresan, Subvisions of ladders are graceful, Indian J. Pure Appl. Math., 23 (1992), 21-23. 